Check for updates

Cite this: RSC Adv., 2018, 8, 42424

Accepted 30th November 2018

DOI: $10.1039 / \mathrm{c} 8 \mathrm{ra03213k}$

rsc.li/rsc-advances

\section{Bipodal dyes with bichromic triphenylamine architectures for use in dye-sensitized solar cell applications $\uparrow$}

\author{
Omar K. Abdi, ${ }^{a}$ Benjamin J. D. Fischer, ${ }^{a}$ Tamara Al-Faouri, ${ }^{a}$ Francis L. Buguis, ${ }^{a}$ \\ Hardeep S. Devgan, ${ }^{a}$ Eduardo Schott, (D) ${ }^{b}$ Ximena Zarate ${ }^{c}$ and Bryan D. Koivisto (D) *a
}

A family of four bipodal triphenylamine-based dyes, three of which incorporate two triphenylamine (TPA) units, have been studied to understand their potential in light-harvesting applications. Compared to previously reported TPA-based dyes, these exhibit improved device performance. Theoretical calculations correlate excited state dipole moments to device efficiency.

\section{Introduction}

Over the last decade, organic light-harvesting dyes have received considerable attention owing to their facile synthesis, tunability and high extinction coefficients when directed towards light-harvesting applications. ${ }^{1-4}$ Organic chromophores driven by intense $\pi-\pi^{*}$ absorption bands tend to have sharp absorptions with minimal effective charge redistribution unless they are outfitted with donor (D) and acceptor (A) units. Incorporation of a $\mathrm{D}-\pi-\mathrm{A}$ motif, however, facilitates low energy intramolecular charge transfer, necessary for charge extraction..$^{5-7}$ While the catalogue of donors, $\pi$-spacers and acceptor groups is large, upon charge extraction, the redox stability of the organic dye is an important consideration. To this end, redox robust triphenylamines (TPAs) have become ubiquitous as building blocks in lightharvesting scaffolds, particularly in the literature relating to dye-sensitized solar cell (DSSC) applications. ${ }^{8-12}$ In addition, TPA dyes have shown themselves to be effective owing to their electron rich nature, redox stability and non-planarity, which mitigates intermolecular dye aggregation. Since the benchmark reports of TPA as a donor in DSSC applications, ${ }^{13-16}$ two champion ( $>10 \%$ efficiency) organic dyes have emerged with this donor motif. ${ }^{17,18}$

To realize further improvements in organic dyes for DSSC applications, three significant challenges must still be addressed. (1) Increased panchromatic absorption into the

${ }^{a}$ Ryerson University, Department of Chemistry \& Biology, 350 Victoria St, Toronto, ON M5B 2K3, Canada. E-mail: bryan.koivisto@ryerson.ca

${ }^{b}$ Departamento de Química Inorgánica, Facultad de Química, Pontificia Universidad Católica de Chile, Avda. Vicuña Mackenna 4860, Santiago, Chile

'Instituto de Ciencias Químicas Aplicadas, Facultad de Ingeniería, Universidad Autonoma de Chile, Llano Subercaseaux 2801, Santiago, Chile

$\dagger$ Electronic supplementary information (ESI) available. See DOI: $10.1039 / \mathrm{c} 8 \mathrm{ra} 03213 \mathrm{k}$
near-IR; (2) improved redox stability and dye lifetime; and (3) improved electronic communication between interfaces ( $\mathrm{TiO}_{2} /$ dye and dye/electrolyte). To address the issue of more broadly absorbing dyes, many studies have explored cosensitization using sensitizers that have complementary absorption envelopes. ${ }^{19,20}$ Cosensitization assumes improvements in the absorption profile that will be able to compensate for attenuated dye concentration; however, this is not often realized.

Another approach for broader absorption profiles has included the design of dyes with multiple chromophores. To this end, we have previously reported modified triphenylamine donors $^{21}$ and bichromic dye motifs. ${ }^{22,23}$ While our previous work has investigated improvements at the dye/electrolyte interface, ${ }^{21}$ in this work, we sought to develop a simple multi-chromic synthetic architecture based exclusively on TPA units.

Herein, we present the following bichromic-bipodal TPA dye family (OA1, OA2, OA3; Fig. 1) and reference compounds OA4 and L1. The bipodal (two cyanoacetic acid anchors) motif has been included to address stability and electronic communication at the semiconductor/dye interface. In addition, incorporation of a second redox-active TPA units seeks to address panchromatic absorption, charge separation and redox robustness within the organic dye motif while creating a $\mathrm{D}-\pi-\mathrm{D}-\pi-\mathrm{A}$ dye system. While multi-donor triarylamine systems have been previously reported, ${ }^{24,25}$ these systems are typically characterized by extensive synthesis and large dye hydrodynamic volumes that can lead to decreased dye loading on the semiconductor surface; however, there have been a family of bipodal dyes reported, that show promising dye architectures. ${ }^{26-29}$ Herein, we present the physicochemical behaviour of a novel family of bichromic/bipodal dyes and offer a rigorous theoretical hypothesis as to why these various dye architectures have significantly better device performance when compared to $\mathbf{L 1}$ (in our hands). 


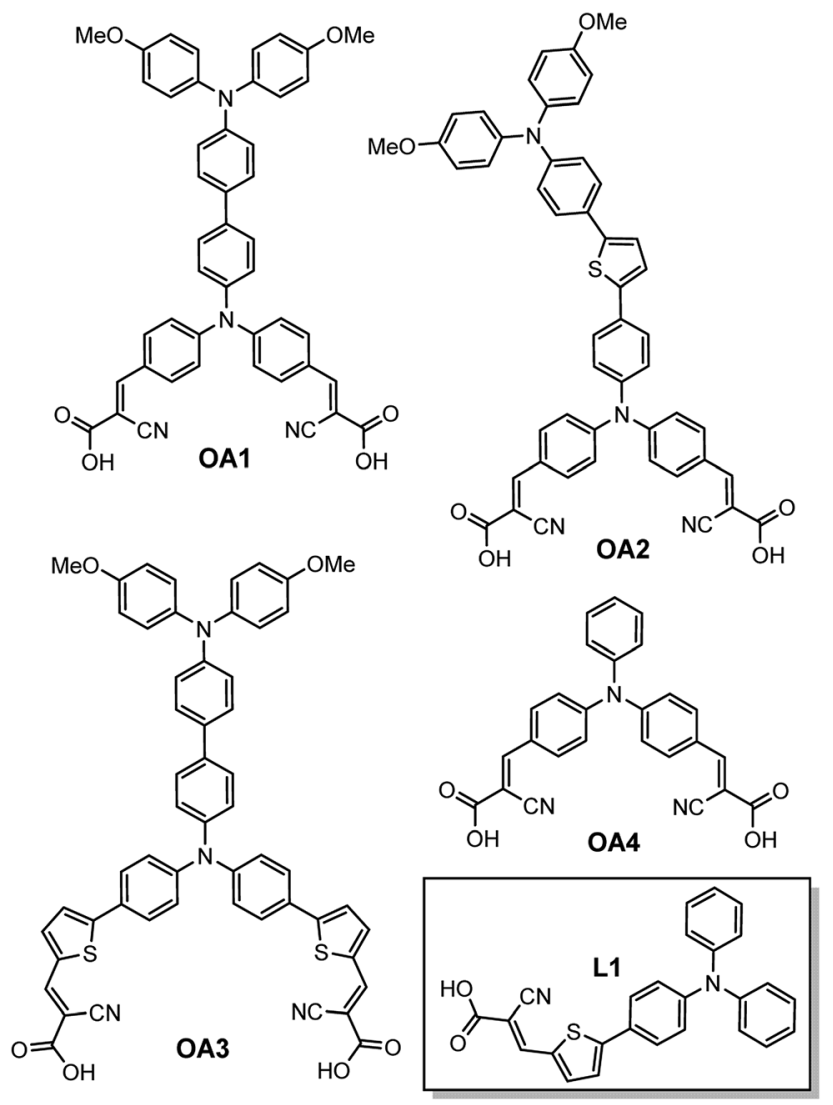

Fig. 1 Bichromic/bipodal dyes OA1, OA2, OA3, and reference dyes OA4 and L1.

\section{Synthesis}

The full synthetic procedures and characterization of these novel dyes, reference compounds (Fig. 1) and precursors can be found in the electronic ESI (Scheme S1†).

The set of dyes OA1, OA2 and OA3 differ in the positioning of the thiophene-bridge or lack thereof (OA1). The intent of this bridge adjustment was to examine the electronic communication between both the anchors and donors, and between the TPA donors themselves; not surprisingly these adjustments had a significant impact on the excited state dipole of the dye (vide infra). To contrast the effectiveness of multiple triphenylamine donor units on the molecular absorption envelope and redox behaviour, we also prepared a monochromic TPA dye OA4 and previously reported $\mathbf{L 1}^{13}$ as reference compounds.

\section{Results and discussion}

To systematically appreciate the behaviour of these dyes, the physicochemical properties are collated and presented in Table 1. The electrochemical and absorption data for OA1, OA2, OA3, OA4 and L1 was collected in DCM solutions, and the UV-Vis solution data is presented in Fig. 2. In addition, physical data of the precursor aldehydes can be found in the ESI (Table S1 and Fig. S2 $\dagger$ ). It should be noted that these dyes do not exhibit any appreciable fluorescence behaviour in solution. As mentioned
Table 1 Physiochemical properties of biopodal dyes

\begin{tabular}{lllll}
\hline & & \multicolumn{3}{l}{$E_{1 / 2}{ }^{b}(\mathrm{~V} v s}$. \\
Dye & $\mathrm{UV} \mathrm{Vis}^{a}, \lambda_{\max } \mathrm{nm}\left(\varepsilon \times 10^{-4} \mathrm{M}^{-1} \mathrm{~cm}^{-1}\right)$ & $\mathrm{NHE})$ & $E_{0-0}{ }^{c}(\mathrm{eV})$ \\
\hline OA1 & $471(3.3)$ & 0.93 & 1.41 & 2.33 \\
OA2 & $422(4.8)$ & 0.98 & 1.33 & 2.45 \\
OA3 & $497(0.5)^{d}$ & 0.89 & 1.20 & 2.05 \\
OA4 & $461(2.6)$ & - & 1.47 & 2.44 \\
L1 & $483(3.2)$ & 1.24 & - & 2.23
\end{tabular}

${ }^{a}$ Low energy visible transitions from UV-Vis in DCM. ${ }^{b}$ Data collected using $0.1 \mathrm{M} \mathrm{NBu}_{4} \mathrm{PF}_{6}$ DCM solutions at $100 \mathrm{mV} \mathrm{s}^{-1}$ and referenced to a $[\mathrm{Fc}] /[\mathrm{Fc}]^{+}$internal standard followed by conversion to NHE; $[\mathrm{Fc}] /\left[\mathrm{Fc}^{+}\right]$ $=+700 \mathrm{mV} v$ s. NHE in DCM. ${ }^{30}{ }^{c}$ Calculated HOMO-LUMO gap based on lowest oxidation potential and low energy absorption. ${ }^{d}$ Lower than expected extinction coefficient is a result of low dye solubility in DCM.

previously, TPA donors show reversible oxidation potentials that can be tuned by appending various electron withdrawing and donating groups.

In all cases, owing to the presence of two redox-active TPA units (with the exception of OA4), the dyes exhibit two reversible oxidation waves/potentials. Based on DFT calculations, the HOMO orbital for all bichromic dyes (OA1, OA2 and OA3) resides on the bisOMeTPA unit (see ESI $\dagger$ ) and the trends for the first measured oxidation potential do show the effect of the thiophene spacer consistent with our previous work. ${ }^{22}$ OA3 has the lowest oxidation potential, because the thiophene spacer proximate to the anchor acts as an electron rich donor mitigating the electron-withdrawing effect of the cyanoacetic acid anchors. In OA2, the presence of the thiophene spacer between the TPA units further stabilizes the HOMO owing to improved conjugation with the more electron deficient TPA unit. In OA1, the facile electronic communication between TPAs no longer exists, and this HOMO stabilization is no longer observed. While it is fairly straightforward to draw trends with the first oxidation potential, the second oxidation potential is more

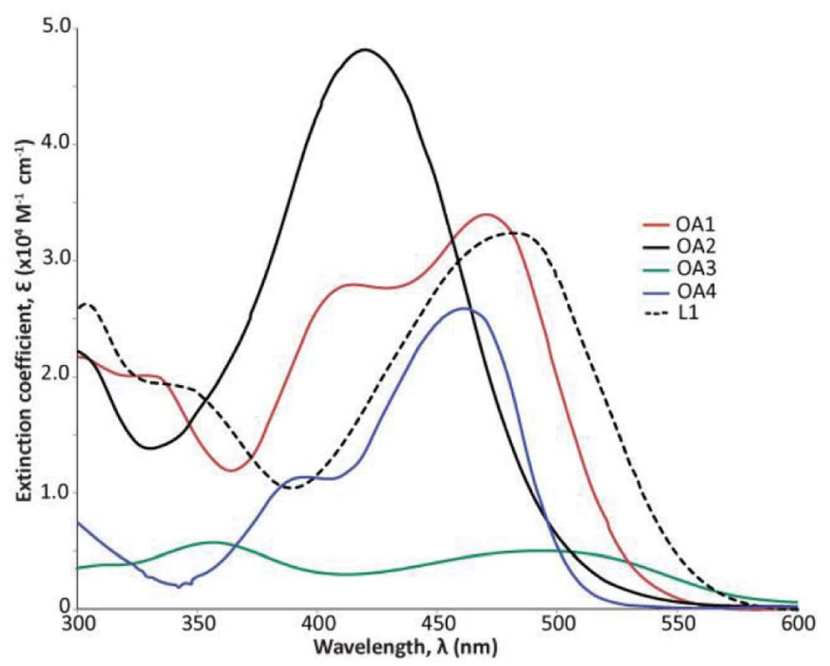

Fig. 2 UV-Vis spectra of dyes OA1, OA2, OA3, OA4 and benchmark L1 in DCM. 
heavily influenced by the reorganizational energy leading to the oxidized product (likely quinoidal in nature).

In the case of the reference bipodal compound OA4, a single oxidation process is observed owing to the presence of only one redox active TPA unit. When comparing the electrochemical behaviour of OA4 to the diformylTPA precursor (see ESI $\dagger$ ), there is only a modest change in the oxidation potential implying that the electron withdrawing effect of an aldehyde and cyanoacetic acid groups are comparable.

The observed changes in the UV-Vis spectra in terms of more broadly absorbing behaviour and extinction coefficient intensity can be rationalized using TD-DFT (see ESI $\dagger$ ). Looking at the absorption profile (Fig. 2) for OA4 refutes the notion that the addition of another donor unit is necessary to create a more broadly absorbing absorption profile. OA4 has two absorption bands when compared to L1, which suggests that the breadth of absorption is imparted due to the addition of a second acceptor unit and not the donor unit. Regardless, a broader absorption envelope is observed for all bisTPA dyes (OA1, OA2 and OA3) when compared to OA4. Like OA4, OA1 exhibits two wellresolved maxima with increasing energy, assigned as the HOMO-1 to LUMO and HOMO to LUMO+2, respectively, see Table S2 (ESI $\dagger$ ). While OA2 has approximately the same crosssection as other dyes, it exhibits a much higher extinction coefficient. Furthermore, in the case of OA2 TDDFT calculations predict that owing to the significant electronic communication between the TPA units, the observed transition emerges from the HOMO-1 to LUMO+1. This transition is closer in energy to the HOMO-LUMO+1 transition and as a result a coalescence of the bichromic absorptions occurs resulting in a hypsochromic shift in the absorption maxima and an increase in the observed extinction coefficient.

The low extinction coefficient for OA3 is consistent with poor solubility in DCM, but TD-DFT data does suggest that a low extinction coefficient is observed because of hindered polarizability owing to the spatial distance between donor and acceptor and the lack of electronic communication between the TPA moieties. Furthermore, as observed for the other dyes, the HOMO-1 to LUMO and HOMO-2 to LUMO+1 transitions dominate the absorption spectrum of OA3, and the HOMOLUMO transition shows also a significant oscillator strength, which would explain the long absorption tail (see Table S2†).

Based on the physical data above, when considering the utility of these dyes for DSSC applications, the HOMO orbital resides at a favourable energy when considering the common electrolytes and regeneration dynamics. ${ }^{31}$ In addition, each of these dyes possess LUMO orbitals situated on the acceptor units, and therefore injection should be kinetically favourable. When compared to benchmark L1, the blue shifted or weaker absorbing profiles of OA1, OA2 and OA3 may manifest in weaker device performance, but the addition of multiple redox active centres may increase the redox robustness of the dye, and could improve electron injection rates. To test these predictions, devices were prepared and theoretical calculations performed to gather further insight.

Multiple devices for each dye were prepared, tested and the data collated in Table 2. Further device characterization (EIS, J-
Table 2 Photovoltaic performance of DSSCs based on bipodal dyes and benchmark $\mathrm{L}^{a}$

\begin{tabular}{llllll}
\hline Dye & $V_{\text {oc }}(\mathrm{V})$ & $J_{\text {sc }}\left(\mathrm{mA} \mathrm{cm}^{-2}\right)$ & FF & $\eta(\%)$ & $N$ \\
\hline OA1 & $0.64 \pm 0.01$ & $3.20 \pm 0.64$ & $0.70 \pm 0.03$ & $2.00 \pm 0.35$ & 7 \\
OA2 & $0.68 \pm 0.01$ & $3.30 \pm 0.43$ & $0.67 \pm 0.05$ & $2.20 \pm 0.23$ & 7 \\
OA3 & $0.58 \pm 0.01$ & $2.70 \pm 0.02$ & $0.72 \pm 0.01$ & $1.69 \pm 0.06$ & 5 \\
OA4 & $0.40 \pm 0.17$ & $0.80 \pm 0.16$ & $0.50 \pm 0.16$ & $0.30 \pm 0.20$ & 5 \\
L1 & $0.52 \pm 0.02$ & $1.18 \pm 0.03$ & $0.58 \pm 0.04$ & $0.53 \pm 0.05$ & 5
\end{tabular}

${ }^{a} \mathrm{~N}$ is the number of simultaneous test cells prepared that makes up the mean and standard deviation. Z1137 $\Gamma / I_{3}{ }^{-}$electrolyte employed: see ESI for complete device fabrication details.

$V$ curves, IPCE) can be found in the ESI. $\dagger$ Satisfyingly, OA1, OA2 and OA3 all substantially outperformed the benchmark L1, largely owing to improved $J_{\mathrm{sc}}$ values. Owing to the bipodal nature of these dyes, solution properties like UV-Vis absorption, are not expected to directly translate to the device performance to when anchored to $\mathrm{TiO}_{2}$ (as shown in the IPCE data Fig. S19, ESI $\dagger$ ). To provide a rational explanation to the obtained performances, quantum chemical calculations were performed on the dyes anchored to a $\mathrm{TiO}_{2}$ cluster model. The molecular orbital energy levels for the three bipodal dyes show that the HOMOs of the free dyes are located under the potential energy of $-4.8 \mathrm{eV}$ of the electrolyte $\Gamma / I_{3}{ }^{-}$. However, in case of the anchored dyes, the energy value is equal to $-4.8 \mathrm{eV}$ or has a bigger value, which in theory would make it less favourable for the dye regeneration after the photooxidation.

On the other hand, using Markus theory, some photovoltaic parameters were evaluated and reported in Table 3 . The free energy of electron injection $\left(\Delta G_{\text {inj }}\right)$, which was computed according to $\Delta G_{\text {inj }}=E_{\text {dye* }}-E_{\mathrm{CB}}$, where $E_{\mathrm{CB}}$ is the reduction potential of the conduction band of $\mathrm{TiO}_{2}(-4.00 \mathrm{eV}) . E_{\text {dye }}$ is the excited-state oxidation potential of the dye, is related to the spontaneous charge transfer from the dyes towards the semiconductor. In this sense, in every case a favorable $\Delta G$ of injection is observed. It is worthy to mention that this parameter is higher for the herein reported dyes than a family of proposed dyes with one TPA as donor, a series of thiophene as bridges and the cyanoacetic acid as anchor motif. ${ }^{32}$ The driving force for charge recombination $\left(\Delta G_{\mathrm{rec}}^{0}\right)$ has been calculated according to $\Delta G_{\mathrm{rec}}^{0}=E_{\mathrm{CB}}-E_{\mathrm{HOMO}}$. As observed, the results for the three systems are very similar, showing that OA1 is the dye that would display the most (although slight) charge recombination when compared with the other two dyes (OA2 and OA3). The light-

Table 3 Theoretical calculations for bipodal dyes ${ }^{a}$

\begin{tabular}{lllllr}
\hline Dye & $-\Delta G_{\text {inj }}$ & $\Delta G_{\text {rec }}$ & LHE & $\mathrm{eV}_{\text {OC }}$ & \multicolumn{1}{c}{$\mu$} \\
\hline OA1 & 1.45 & 1.07 & 0.90 & 1.07 & 11.36 \\
OA2 & 1.77 & 1.01 & 0.91 & 1.06 & 11.77 \\
OA3 & 1.16 & 1.03 & 0.93 & 1.01 & 4.21
\end{tabular}

${ }^{a}$ Calculated negative free energy of the injection $\left(-\Delta G_{\mathrm{inj}},[\mathrm{eV}]\right)$, recombination free energy $\left(\Delta G_{\mathrm{rec}},[\mathrm{eV}]\right)$, light-harvesting efficiency (LHE, $[\mathrm{eV}]$ ) for the lowest singlet excited state, the open-circuit voltage $\left(\mathrm{eV}_{\mathrm{OC}}[\mathrm{eV}]\right)$ and dipole moments $(\mu)$ for the dye. 
harvesting efficiency (LHE) has been computed according to LHE $=1-10^{f}$, where $f$ is the oscillator strength of the dye related to the $\lambda_{\max }$. This parameter can be interpreted as the light absorbance of the dye-sensitized $\mathrm{TiO}_{2}$ film and should be as high as possible in order to maximize the photocurrent response. Finally, the $\mathrm{eV}_{\mathrm{oc}}\left(\mathrm{eV}_{\mathrm{OC}}=E_{\mathrm{LUMO}}-E_{\mathrm{CB}}\right)$ represents the maximum voltage available from a solar cell which occurs at zero current. Although, there are some arguments about this approximation there is good agreement between the experimental and calculated values, where OA1 shows the biggest $V_{\text {oc }}$ value, while OA3 shows the smallest value.

To further appreciate the improved device performance, recent electrochemical studies suggest that the interfacial dipole moment directed to the semiconductor contributes to better understanding of performance when considering electron injection processes and the generated voltage. ${ }^{32}$ The magnitude of the dipole moments for the free dyes shown in Table 3, indicates that OA2 shows the biggest value among all the studied dyes and its dipole moment vector is directed to the anchor motif. This property is also found for OA1 and OA3 as can be seen in Fig. 3, and correlates well to the observed device efficiencies.

The oxidized states of the dyes in a DSSC after the electron photo-injection play an important role because: (1) this oxidized dye is regenerated to the ground state by the electrolyte that commonly are $\Gamma / I_{3}{ }^{-}(4.60 \mathrm{eV})$ or the $\mathrm{Co}^{\mathrm{III} / \mathrm{II}}(5.0 \mathrm{eV})$ redox couples, among others, also (2) there exist intermolecular hole hopping, and lateral recombination can occur from one dye to a second dye in the oxidized state and (3) a charge recombination between the charged semiconductor and the oxidized dye $\mathrm{TiO}_{2}\left(\mathrm{e}^{-}\right) /$dye $e^{+}$is possible; where the electron moves back to the parent dye, reducing the efficiency of the photo-conversion.

For this latter case, it is stated that the localization of the hole over the donor fragment after photo-injection generates a charge separation, which leads to decreased probability of
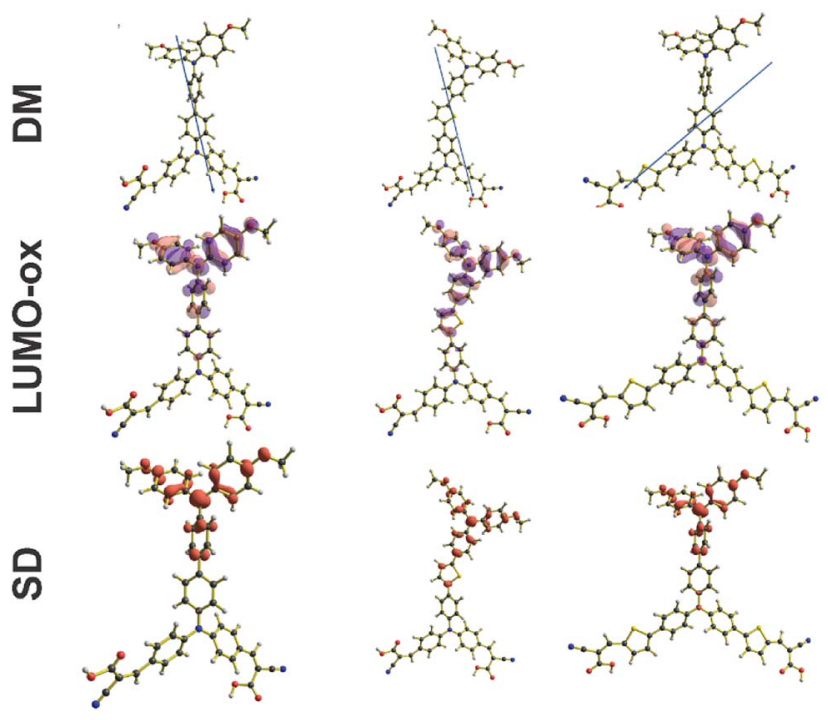

Fig. 3 Free dyes dipole moment (DM), LUMO (LUMO-OX) isosurfaces and spin density (SD) of the oxidized species. interfacial electron back-transfer. The isosurface and spin density of the LUMO of the oxidized state (Fig. 3), shows the dye after electron removal. Therefore, this isosurface or spin density represents the hole generated after charge photoinjection into the semiconductor. Fig. 3 shows the hole localization for OA1, OA2 and OA3. It is observed that in every case the hole is localized over the donor portion of the molecule. This behaviour is favourable for dye regeneration as there is substantial charge separation after electron injection.

\section{Conclusions}

A set of bipodal multichromic dyes, OA1, OA2 and OA3 have been synthesized and studied. In the absence of device data and theoretical calculations, these dyes may not have the physicochemical properties that would suggest superior performance when compared to benchmark L1; however, this motif shows remarkable promise for light-harvesting and provides further insight and serves as a guiding platform for organic dye design.

\section{Conflicts of interest}

There are no conflicts to declare.

\section{Acknowledgements}

BDK would like to acknowledge NSERC and Ryerson University for their financial support. ESV and XZB would like to acknowledge, Fondap/serc chile/15110019 and Fondecyt 1161416 and 1180565, respectively.

\section{Notes and references}

1 A. Mishra, M. K. R. Fischer and P. Bauerle, Angew. Chem., Int. Ed., 2009, 48, 2474-2499.

2 J. Roncali, Acc. Chem. Res., 2009, 42, 1719-1730.

3 A. Mishra and P. Bauerle, Angew. Chem., Int. Ed., 2012, 51, 2020-2067.

4 Y. Sun, G. C. Welch, W. L. Leong, C. J. Takacs, G. C. Bazan and A. J. Heeger, Nat. Mater., 2012, 11, 44-48.

5 X. B. Sun, Y. Q. Liu, X. J. Xu, C. H. Yang, G. Yu, S. Y. Chen, Z. H. Zhao, W. F. Qiu, Y. F. Li and D. B. Zhu, J. Phys. Chem. $B$, 2005, 109, 10786-10792.

6 R. Jose, A. Kumar, V. Thavasi, K. Fujihara, S. Uchida and S. Ramakrishna, Appl. Phys. Lett., 2008, 93, 023125.

7 V. Thavasi, V. Renugopalakrishnan, R. Jose and S. Ramakrishna, Mater. Sci. Eng., R, 2009, 63, 81-99.

8 Y. Ooyama and Y. Harima, Eur. J. Org. Chem., 2009, 29032934.

9 A. Hagfeldt, G. Boschloo, L. Sun, L. Kloo and H. Pettersson, Chem. Rev., 2010, 110, 6595-6663.

10 K. C. D. Robson, B. D. Koivisto, A. Yella, B. Sporinova, M. K. Nazeeruddin, T. Baumgartner, M. Grätzel and C. P. Berlinguette, Inorg. Chem., 2011, 50, 5494-5508.

11 R. K. Kanaparthi, J. Kandhadi and L. Giribabu, Tetrahedron, 2012, 68, 8383-8393.

12 M. Liang and J. Chen, Chem. Soc. Rev., 2013, 42, 3453-3488. 
13 D. P. Hagberg, T. Marinado, K. M. Karlsson, K. Nonomura, P. Qin, G. Boschloo, T. Brinck, A. Hagfeldt and L. Sun, J. Org. Chem., 2007, 72, 9550-9556.

14 S. Hwang, J. H. Lee, C. Park, H. Lee, C. Kim, C. Park, M.-H. Lee, W. Lee, J. Park, K. Kim, N.-G. Park and C. Kim, Chem. Commun., 2007, 4887-4889.

15 M. Liang, W. Xu, F. Cai, P. Chen, B. Peng, J. Chen and Z. Li, J. Phys. Chem. C, 2007, 111, 4465-4472.

16 W. Xu, B. Peng, J. Chen, M. Liang and F. Cai, J. Phys. Chem. C, 2008, 112, 874-880.

17 C.-P. Hsieh, H.-P. Lu, C.-L. Chiu, C.-W. Lee, S.-H. Chuang, C.-L. Mai, W.-N. Yen, S.-J. Hsu, E. W.-G. Diau and C.-Y. Yeh, J. Mater. Chem., 2010, 20, 1127-1134.

18 W. D. Zeng, Y. M. Cao, Y. Bai, Y. H. Wang, Y. S. Shi, M. Zhang, F. F. Wang, C. Y. Pan and P. Wang, Chem. Mater., 2010, 22, 1915-1925.

19 Y. Hong, J.-Y. Liao, D. Cao, X. Zang, D.-B. Kuang, L. Wang, H. Meier and C.-Y. Su, J. Org. Chem., 2011, 76, 8015-8021.

20 V. Leandri, R. Ruffo, V. Trifiletti and A. Abbotto, Eur. J. Org. Chem., 2013, 6793-6801.

21 C. Bonnier, D. D. Machin, O. K. Abdi, K. C. Robson and B. D. Koivisto, Org. Biomol. Chem., 2013, 11, 7011-7015.

22 C. Bonnier, D. D. Machin, O. Abdi and B. D. Koivisto, Org. Biomol. Chem., 2013, 11, 3756-3760.
23 M. Yousaf, E. Schott, A. Lough and B. D. Koivisto, RSC Adv., 2015, 5, 57490-57492.

24 C.-H. Yang, S.-H. Liao, Y.-K. Sun, Y.-Y. Chuang, T.-L. Wang, Y.-T. Shieh and W.-C. Lin, J. Phys. Chem. C, 2010, 114, 21786-21794.

25 K. Oum, O. Flender, P. W. Lohse, M. Scholz, A. Hagfeldt, G. Boschloo and T. Lenzer, Phys. Chem. Chem. Phys., 2014, 16, 8019-8029.

26 M. B. Desta, S. Chaurasia and J. T. Lin, Dyes Pigm., 2017, 140, 441-451.

27 K. D. Seo, B. S. You, I. T. Choi, M. J. Ju, M. You, H. S. Kang and H. K. Kim, J. Mater. Chem. A, 2013, 1, 9947-9953.

28 K. D. Seo, B. S. You, I. T. Choi, M. J. Ju, M. You, H. S. Kang and H. K. Kim, ChemSusChem, 2013, 6, 2069-2073.

29 K. D. Seo, B. S. You, I. T. Choi, M. J. Ju, M. You, H. S. Kang and H. K. Kim, Dyes Pigm., 2013, 99, 599-606.

30 C. M. Cardona, W. Li, A. E. Kaifer, D. Stockdale and G. C. Bazan, Adv. Mater., 2011, 23, 2367-2371.

31 X. Zarate, M. Saavedra-Torres, A. Rodriguez-Serrano, T. Gomez and E. Schott, J. Comput. Chem., 2018, 39, 685-698.

32 K. Hu, K. C. D. Robson, E. E. Beauvilliers, E. Schott, X. Zarate, R. Arratia-Perez, C. P. Berlinguette and G. J. Meyer, J. Am. Chem. Soc., 2014, 136, 1034-1046. 